\title{
Troponin I Type 2 Measurement
}

National Cancer Institute

\section{Source}

National Cancer Institute. Troponin I Type 2 Measurement. NCI Thesaurus. Code

C127636.

The determination of the amount of toponin I type 2 present in a sample. 